\title{
A survey of the weakest-field magnetic Ap stars: discovery of a threshold magnetic field strength?
}

\author{
M. Aurière ${ }^{1}$, J. Silvester ${ }^{2}$, G.A. Wade $^{2}$, S. Bagnulo ${ }^{3}$, J.-F. Donati ${ }^{1}$, \\ N. Johnson ${ }^{2}$, F. Lignières ${ }^{1}$, J.D. Landstreet ${ }^{4}$, T. Lüftinger ${ }^{5}$, \\ D. Mouillet ${ }^{1}$, F. Paletou ${ }^{1}$, P. Petit ${ }^{6}$ and S. Strasser ${ }^{7}$ \\ ${ }^{1}$ Observatoire Midi Pyrénées, France \\ ${ }^{2}$ Department of Physics, Royal Military College of Canada \\ ${ }^{3}$ European Southern Observatory, Chile \\ ${ }^{4}$ University of Western Ontario, Canada \\ ${ }^{5}$ Universitat Wien Institut für Astronomie, Austria \\ ${ }^{6}$ Max Planck Institut für Astrophysik, Germany \\ ${ }^{7}$ University of Calgary, Canada
}

\begin{abstract}
We are conducting a magnetic survey of a sample of about 30 spectroscopically identified Ap stars, with weak or previously undetected magnetic fields. For 28 studied stars, we have obtained 25 detections of Stokes $V$ Zeeman signatures. Our results suggest that all Ap stars are magnetic. Further there may exist a minimum field strength for which Ap-type characteristics are produced.
\end{abstract}

Keywords. Methods: data analysis, techniques: polarimetric, stars: magnetic fields

\section{Introduction}

Although thousands of chemically peculiar (CP) and Ap stars are catalogued (Renson et al. 1991), only about 210 Ap stars have magnetic field measurements (Romanyuk 2000). The catalogue of magnetic CP stars by Romanyuk (2000) contains 117 of 211 stars (55\%) with maximum unsigned longitudinal fields larger than $1 \mathrm{kG}$. On the other hand, according to Bohlender \& Landstreet (1990), the median rms longitudinal magnetic field of Ap stars (based on a magnitude-limited sample observed by Borra \& Landstreet 1980) is approximately $300 \mathrm{G}$ (the largest one they report is only $710 \mathrm{G}$ ). In the catalogue of Bychkov et al. (2003) the distribution of the averaged magnetic field strength is described by a decreasing exponential function above $100 \mathrm{G}$, which again implies that weak-field Ap stars represent the majority of their class. Thus the weak part of the magnetic distribution in Ap stars is unknown and one may even ask if there is there is a minimum magnetic field needed for making an Ap star (Glagolevskij \& Chountonov 2002). To improve our knowledge of these stars and to be able to make an unbiased investigation of Ap star magnetic fields, we have undertaken the study of a sample of about 30 spectroscopically identified Ap stars (selected from the HD catalogue), but with weak or previously undetected magnetic fields.

\section{Method}

Observations were obtained during 5 runs from June 2001 to February 2004 using the $\mathrm{MuSiCoS}$ spectropolarimeter attached to the Bernard Lyot telescope (TBL) at the Ob- 


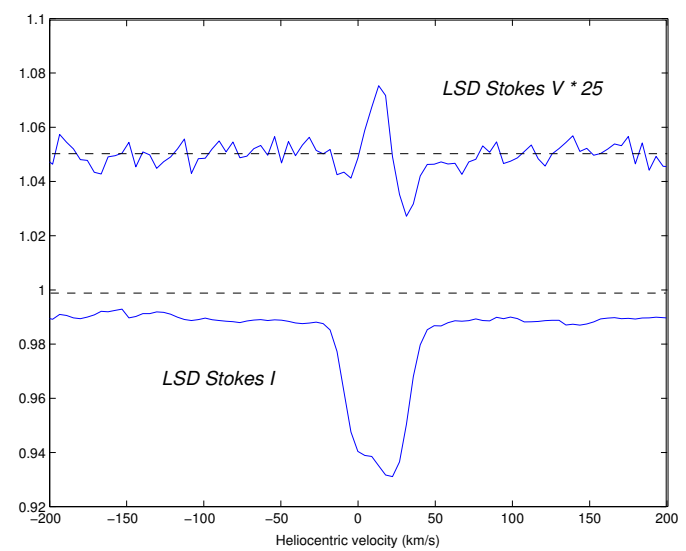

Figure 1. Stokes $I$ and Stokes $V$ LSD Zeeman signatures for the weak-field magnetic Ap star 43 Cas (HD 10221). Notice the easily-detected Stokes $V$ Zeeman signature, although at a phase when the longitudinal magnetic field is only $93 \pm 32 \mathrm{G}$.

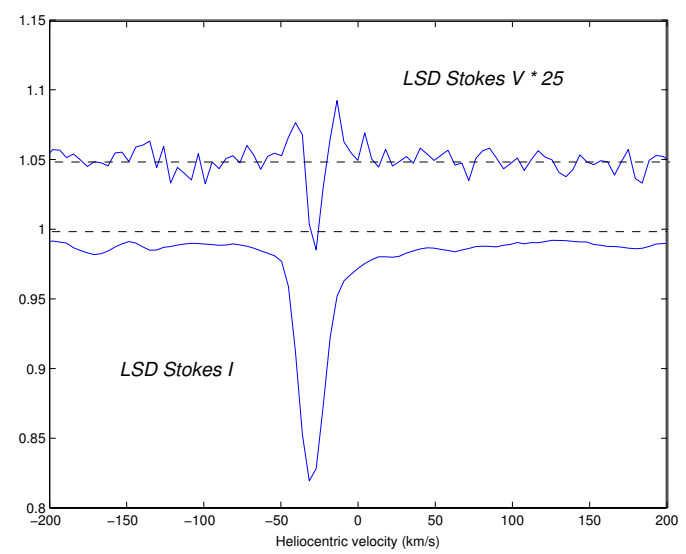

Figure 2. Stokes $I$ and Stokes $V$ LSD Zeeman signatures for the weak-field magnetic Ap star $65 \mathrm{UMa}$, at a phase when the longitudinal magnetic field is only $-43 \pm 26 \mathrm{G}$.

servatoire du Pic du Midi. The aim of our study is to detect circular polarisation which is characteristic of the longitudinal Zeeman effect. For this we used the Least Squares Deconvolution (LSD) procedure (Donati et al. 1997). This method enables the "averaging" of several hundreds of lines and thus to obtain Stokes $I$ and Stokes $V$ profiles with greatly improved $S / N$ ratios. LSD gives us a single quantitative criterion for detection of Stokes $V$ polarisation: we perform a statistical test in which the reduced $\chi^{2}$ statistic is computed for the Stokes $V$ profile, both inside and outside the spectral line (Donati et al 1997). The statistics are then converted to detection probabilities and the probabilities are assessed to determine if we have definite detection (DD, false alarm probability $<10^{-5}$ ), marginal detection (MD, false alarm probability $>10^{-5}$ and $<10^{-3}$ ), or no detection. We also computed mean longitudinal magnetic fields $B_{\mathrm{z}}$ for the stars in our survey, Figures 1 through 3 illustrate that we can obtain strong Stokes V polarisation detections, and thus significant magnetic field detection, even if $B_{\mathrm{z}}$ is null at the observed phase. 


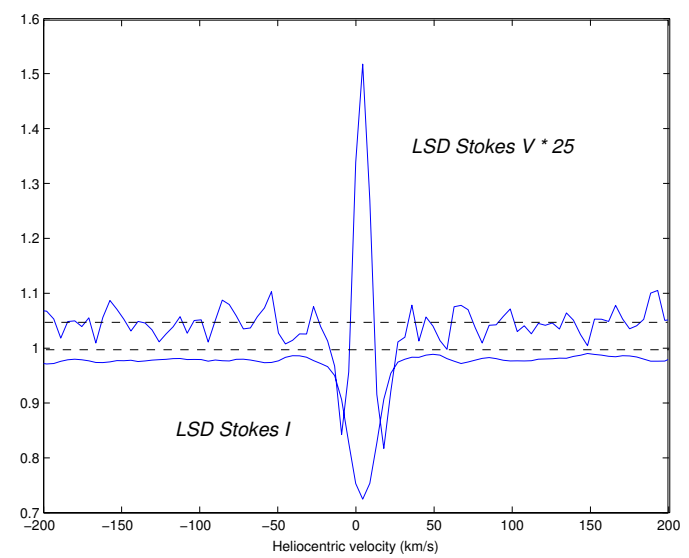

Figure 3. Stokes $I$ and Stokes $V$ LSD Zeeman signatures for the magnetic Ap star HD 43819. Notice the very strong Zeeman signature, although at a phase when the longitudinal magnetic field is only $-72 \pm 39 \mathrm{G}$.

Table 1. Maximum $\left|B_{z}\right|$ observed for definitively detected stars of our survey (results are currently improving with new observations and new reductions). Note that the majority of these stars are of early A/late B spectral type; hence this survey complements the survey of cool Ap stars reported by Johnson et al. (2005).

\begin{tabular}{rccccc}
\hline Name & HD & Spec.Type & \# Obs & Max. Obs. $\left|B_{z}\right|(\mathrm{G})$ & Error $(\mathrm{G})$ \\
\hline HN And & 8441 & A2p & 1 DD (1) & 65 & 29 \\
43 Cas & 10221 & A0sp & 3 DD (6) & 93 & 32 \\
$\iota$ Cas & 15089 & A5p & 2 DD (2) & 285 & 140 \\
& 15144 & A6Vsp & 2 DD (2) & 648 & 19 \\
9 Tau & 22374 & A2p & 1 DD (2) & 459 & 32 \\
56 Tau & 27309 & A0sp & 5 DD (5) & 649 & 79 \\
& 37687 & B8 & 1 DD (1) & 760 & 165 \\
& 40711 & A0 & 1 DD (1) & 492 & 75 \\
& 43819 & B9IIIsp & 6 DD (6) & 770 & 79 \\
15 Cnc & 68351 & B9sp & 1 DD (7) & 264 & 62 \\
3 Hya & 72968 & A1spe & 8 DD (8) & 371 & 26 \\
45 Leo & 90569 & A0sp & 8 DD (8) & 538 & 45 \\
& 94427 & A5 & 1 DD (4) & 194 & 47 \\
& 96707 & F0p & 1 DD (6) & 119 & 58 \\
65 Uma & 103498 & A1spe & 7 DD (8) & 160 & 21 \\
21 Com & 108945 & A2pvar & 7 DD (7) & 256 & 52 \\
& 138633 & F0 & 1 DD (2) & 246 & 48 \\
$\omega$ Her & 148112 & B9p & 4 DD (5) & 234 & 43 \\
10 Aql & 176232 & F0spe & 3 DD (3) & 337 & 15 \\
19 Lyr & 179527 & B9sp & 2 DD (5) & 329 & 92 \\
4 Cyg & 183056 & B9sp & 2 DD (2) & 186 & 134 \\
& 204411 & A6pe & 1 DD (1) & 30 & 11 \\
$\kappa$ Psc & 220825 & A0p & 2 DD (2) & 212 & 86 \\
\hline
\end{tabular}

\section{Results}

For the 28 stars observed to date, 25 are exhibit a significant circular polarisation in their spectral lines (although of only marginally significance in two cases). For a majority 
of the sample, a detection was obtained during the first observation. For some objects, obtaining one positive detection required several observations. This can be due to phase effects and of course to meteorological conditions and/or not having large enough signal to noise ratio. For three of our stars with a sufficiently large number of measurements and suitable phase sampling, and having well-determined periods (3 Hya, $21 \mathrm{Com}, 45 \mathrm{Leo}$ ), dipolar oblique rotator models have been determined. We find that geometry alone can explain the weakness of the measured fields, at least in some cases. Table 1 shows the maximum unsigned longitudinal magnetic field measured presently for each star of our survey which is definitively detected (DD). These values are rather high, and when one takes into account that the polar field strength in an Oblique Rotator Model (Preston 1967 ) is greater than 3 times this value, the suggested polar field strengths must be at least several hundred G. This result may indicate that there is a minimum field strength for which Ap-type characteristics are produced. This minimum strength appears to be of order the photospheric equipartition field (around $250 \mathrm{G}$ for a main sequence A0 star). The remarkable detection rate obtained in our survey strongly suggests that all Ap stars having "magnetic" behaviour (Preston 1974, i.e., essentially all stars classified spectroscopically as Ap/Bp) actually harbour magnetic fields of at least a few hundred G. In other words, all Ap stars are magnetic stars and there may well be a threshold field strength for obtaining this behaviour.

\section{References}

Bohlender D.A. \& Landstreet J.D., 1990, MNRAS 247, 606

Borra E.F. \& Landstreet J.D., 1980, ApJS 42, 421

Bychkov V.D., Bychkova L.V., Madej J., 2003, A\&A 407, 631

Donati, J.-F., Semel, M., Carter, B.D., Rees, D.E., Cameron, A.C., 1997, MNRAS, 291, 658-682

Glagolevskij Y.V. \& Chuntonov G.A., 2002, Astrophysics 45, 408

Johnson, N, Wade, G. A., Baecasis, M., et al. 2005 These proceedings, EP2

Preston, G. W., 1967, ApJ 150, 547

Preston W., 1974, ARAESA 12, 257

Renson P., Gerbaldi M., Catalano F. A., 1991, A\&\&S 89, 429

Romanyuk I.I., 2000, in "Magnetic fields of chemically peculiar and related stars", Eds Y.V. Glagolevskij \& I.I. Romanyuk (Moscow), 18 and www.sao.ru/ ${ }^{\sim}$ dkudr 